\title{
Opioidtoleranz führt zu Wirkverlust von Lokalanästhetika
}

Liu Q, Gold MS, Opioid-induced Loss of Local Anesthetic Potency in the Rat Sciatic Nerve. Anesthesiology 2016; $125: 755$ - 764

Die zusätzliche Gabe von Lokalanästhetika (LA) gehört nach vielen chirurgischen Eingriffen zum Standard der Schmerztherapie: Sie hilft bei der Einsparung von Opioiden und erlaubt eine schnellere Rehabilitation und Entlassung aus der Klinik. Nun gibt es aber zunehmend Hinweise darauf, dass bei Patienten mit regelmäßiger Opioideinnahme die LA nur vermindert oder gar nicht wirken. Mediziner aus Pittsburgh haben sich das näher angesehen.

Qing Liu und Michael Gold sind dabei dem Verdacht nachgegangen, dass die Opioide bei chronischer Einnahme zu Veränderungen am peripheren Nerven führen, die für diesen Verlust an analgetischer Potenz verantwortlich sind. Dazu haben sie an Ratten verschiedene Versuche durchgeführt. Die Tiere erhielten im 1. Schritt

- 1-malig Morphin $10 \mathrm{mg} / \mathrm{kg}$ s.c. oder

- 7 Tage nacheinander jeweils $10 \mathrm{mg} / \mathrm{kg}$ Morphin s.c.

Anschließend untersuchten die Wissenschaftler am isolierten $\mathrm{N}$. ischiadicus der jeweiligen Tiere die Wirkung von Lidocain auf die Summenaktionspotenziale (SAPs).Dabei fanden sie, dass

- die 1-malige Gabe von Morphin die Potenz eines Lidocain-Nervenblocks nicht beeinträchtigte, aber

- bei den Ratten mit 7-maliger Gabe die EC50 von Lidocain (die Dosis, die 50\% des SAPs blockierte) ca. 3-mal so hoch lag wie bei den nicht mit Morphin behandelten Ratten.

An weiteren Tieren untersuchten die Forscher nun verschiedene Morphindosen und entdeckten, dass

- das Ausmaß der SAP-Verminderung dosisabhängig war und am geringsten bei der Gabe von $7 \times 3 \mathrm{mg} / \mathrm{kg}$ Morphin,

- moderat bei $7 \times 5 \mathrm{mg} / \mathrm{kg}$ und

- am höchsten bei $7 \times 10 \mathrm{mg} / \mathrm{kg}$.

Dabei begann dieser Lidocain-Wirkverlust bereits nach der 2. Morphingabe und nahm im Verlauf bis Tag 7 immer weiter zu.
Wurde nach den 7 Tagen die Morphingabe beendet, war

- die Schmerzempfindung der Tiere (Wegziehen der Pfote bei Hitzeschmerzreiz) 7 Tage nach der letzten Injektion vergleichbar zu der vor der 1. Morphingabe und blieb bei weiteren Tests 21 und 35 Tage nach der letzten Injektion so erhalten,

- der Verlust der lokalanästhetischen Potenz von Lidocain dagegen blieb unverändert bestehen und war auch 35 Tage nach der letzten Injektion ebenso stark wie unmittelbar nach der 7-tägigen Injektionsphase.

Schließlich wollten die Mediziner noch wissen, ob und wie dieser Wirkverlust von Lidocain möglicherweise verhindert werden konnte. Dazu verabreichten sie einer weiteren Gruppe von Ratten

- 7 Tage nacheinander jeweils 10 mg/kg Morphin s.c. plus Naloxon $1 \mathrm{mg} / \mathrm{kg}$ i.p.oder

- 7 Tage nacheinander jeweils 10 mg/kg Morphin s.c. plus Naloxon-Methiodid $1 \mathrm{mg} / \mathrm{kg}$ s. c.

Dabei zeigte sich, dass zwar

- das liquorgängige Naloxon,

- nicht aber das nicht ins ZNS penetrierende Naloxon-Methiodid

den Wirkverlust von Lidocain durch Morphin verhindern konnte.

\section{FAZIT}

Wenn sich diese Daten bestätigen, hat das für die tägliche Praxis der postoperativen Schmerztherapie erhebliche Folgen, so die Autoren. Bei Patienten mit regelmäßiger Opioideinnahme, auch geringer Dosen, wäre für eine ausreichende Wirkung von LA eine deutlich höhere Dosis notwendig - was wiederum das Risiko einer systemischen Toxizität der LA erhöht. Und es wird nicht ausreichen, einfach präoperativ das Opioid abzusetzen, denn der Wirkverlust der LA scheint immerhin > 1 Monat anzuhalten.

\section{Dr. Elke Ruchalla, Bad Dürrheim}

\section{Kommentar}

In der perioperativen schmerzmedizinischen Versorgung werden Patienten mit Opioidmissbrauch in der Vorgeschichte bevorzugt durch regionale Analgesieverfahren behandelt. Dabei soll die Schmerzlinderung durch zentrale oder periphere Lokalanästhesien erfolgen, um Opioide nach Bedarf bei diesen Patienten zu vermeiden. Klinische Beobachtungen sprechen jedoch dafür, dass Lokalanästhestika gerade bei diesen Patienten unzuverlässig sein könnten [1 4]. Diese Hinweise haben die Autoren veranlasst, in einem Experiment den Einfluss von Morphingaben in unterschiedlichen Dosierungen und über verschiedene Zeiträume auf die Effektivität von Lokalanästhetika zu analysieren.

Die Autoren beschreiben eine dosisabhängige Entwicklung von Opioidtoleranz und damit verbunden eine Hemmung der Wirkung von Lidocain auf spannungsabhängige Natriumkanäle: Die Hemmung der Aktionspotenziale wurde durch Morphin vermindert. Dieser Effekt konnte durch die gleichzeitige Gabe von Naloxon vermieden werden. Der Effekt hielt mit 35 Tagen über die Dauer der induzierten Opiodtoleranz hinaus an. 
In der Klinik tritt dieser Effekt nicht immer auf. Viele Patienten mit einem Opioidabusus profitieren in der postoperativen Phase von regionalen Analgesieverfahren. Bei Non-Respondern könnte die vorliegende Untersuchung jedoch erklären, dass nicht eine insuffiziente Technik, sondern die verminderte Wirksamkeit von Lokalanästhetika bei Patienten mit Opioidmissbrauch zu einem Versagen der regionalen Verfahren führen. Systematische Analysen zur perioperativen Schmerztherapie sind v.a. auf die adäquate Substitution der Opioidmedikation gerichtet [5]. Daher sollte bei Patienten, die durch ein regionales Analgesieverfahren nicht angemessen behandelt werden können, die postoperative Schmerztherapie durch Anpassung der Nicht-Opioidanalgetika und der Opioide erfolgen

Die in dem beschriebenen Experiment verwendeten Dosierungen lagen bei 3$10 \mathrm{mg} / \mathrm{kg}$ Körpergewicht. Diese Dosis oder äquivalente Mengen anderer Opioide können bei Opioid- abhängigen Patienten gefunden werden. In der Behandlung chronischer Schmerzen spielen sie eher eine untergeordnete Rolle. Dennoch begegnen uns in der Praxis der Behandlung von Patienten mit chronischen Schmerzen regelmäßig Menschen mit chronischer Opioideinnahme, die eigentlich auf keine Form der Analgesie mit einer zufriedenstellenden Schmerzkontrolle reagieren. Die Patienten berichten nicht selten, dass sie Opioide brauchen, weil sonst alles noch schlimmer werden könne. Können Opioide selbst eine chronische Schmerzerkrankung auslösen oder unterhalten? Die vorliegenden Daten unterstützen die Vermutung, dass die chronische Einnahme von Opioiden eine Neuropathie induzieren kann, die ihrerseits auf dem Boden einer Veränderung der Funktion und Struktur von spannungsabhängigen $\mathrm{Na}$ triumkanälen die Wahrnehmung von Schmerzen steigern und somit selbst chronische Schmerzen auslösen kann.

Auch wenn dies zunächst eine nicht wissenschaftlich gesicherte Übertragung der vorliegenden experimentellen Daten auf Patienten mit einer chronischen Opioidmedikation darstellt, wird deutlich, dass die Bedeutung der Untersuchung weit über die Behandlung von Opioidabhängigen Patienten hinausgeht und unser Verständnis zum Nutzen-Risiko-Verhältnis des Einsatzes von Opioiden bei chronischen Schmerzen schärfen kann.
Der Autor

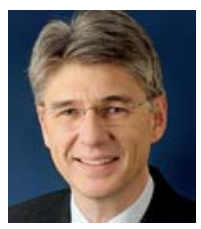

PD Dr. med. Markus

Gehling, Praxis

für Schmerzmedizin, Kassel

Literatur

[1] Liu Q et al. Impact of peripheral nerve block with low dose local anesthetics on analgesia and functional outcomes following total knee arthroplasty: a retrospective study. Pain Med 2015; 16: 998 - 1006

[2] Zywiel MG et al. Chronic opioid use prior to total knee arthroplasty. J Bone Joint Surg Am, 2011; 93: 1988 - 1993

[3] Vosoughian M et al. The duration of spinal anesthesia with $5 \%$ lidocaine in chronic opium abusers compared with nonabusers. Anesth Analg 2007; 105: 531 - 533

[4] Hashemian AM et al. Effectiveness of local anesthesia with lidocaine in chronic opium abusers. J Emerg Trauma Shock 2014; 7: 301 304

[5] Alizadeh S et al. Post-operative Analgesia in Opioid Dependent Patients: Comparison of Intravenous Morphine and Sublingual Buprenorphine. Addict Health 2015; 7: 60-65 